\title{
Experimental Investigation of Polymer Adsorption-Induced Permeability Reduction in Low Permeability Reservoirs
}

\author{
Hyemin Park, Jinju Han, Minkyu Lee, Youngho Jang and Wonmo Sung* \\ Department of Natural Resources and Environmental Engineering, Hanyang University, Seoul, Republic of Korea
}

\begin{abstract}
The polymer retention during polymer flooding causes reduction in pore throat size which can be detrimental to oil recovery. Especially, in low permeability reservoir unlikely in high permeability system, EOR effect of polymer flooding would not excel comparing to water flooding because of the polymer adsorption onto grain surface. This phenomenon is more significant as higher concentration of polymer solution is injected. In this study, we investigated oil recovery process during polymer flooding for a quarter of 2D 5-spot pattern of low permeability sandstone slab, in which the injected polymer solution flows in different shear rates corresponding to streamlines. The results showed that the ultimate recovery was 1.9 times higher in concentration of $1,000 \mathrm{ppm}$ case than $0 \mathrm{ppm}$. The polymer adsorption layer formed during the flooding reduced the effective permeability of $1,000 \mathrm{ppm}$ polymer solution. Therefore, it facilitated the oil flow and the stage of water-cut reaching $99 \%$ was slackened off.
\end{abstract}

Keywords: Polymer retention; Low permeability reservoirs; Permeability; Polymer adsorption

\section{Introduction}

Polymer flooding is a mature technology of chemical EOR method that increases the sweep efficiency and the oil recovery by injecting viscous polymer solution. However, polymer retention in porous media such as adsorption onto grain surface and mechanical entrapment causes the reduction in polymer slug and the mobility reduction efficiency at the location away from the injection well can be deteriorated. Meanwhile, the phenomena of adsorbed polymer layer and mechanical entrapment can make the effect of preventing excessive water production. To understand the mechanisms that enable water permeability reduction, several experimental studies have investigated the resulting effect of polymer adsorption [1-5].

The previous researches indicate a selective action of the polymer with a significant reduction in the relative permeability to water with respect to the relative permeability to oil. Cordova et al. [6] showed polymer adsorption reduced the water permeability regardless of the wettability and relatively facilitated the oil flow. Barreau et al. [7] used capillary tube filled with porous medium in the polymer flooding experiment. They yielded the thickness of polymer adsorption layer with the effective permeability change and found out that the adsorption layer could affect the fluid flow. Chauveteau et al. [8] revealed that when polymer is injected at low shear rates, the adsorbed layer thickness does not depend on the injection rate. When the injection occurs at higher shear rates, the adsorbed layer thickness increases slowly up to some maximum values, increasing with the injection rate. Although the polymer adsorption plays a significant role in relative permeability modification to control the water production, it results in permeability reduction of porous media.

In this work, we attempted to comprehend the effect of polymer adsorption on reducing the water production and enhancing the oil recovery. Also, we intended to explain how the effect of reduction in the relative permeability to water conflicted with the decrease in pore throat size by polymer adsorption layer, therefore, affected to EOR efficiency with the polymer concentration. This experiment has been conducted for two dimensional 5-spot pattern of low permeable sandstone plate. The flow velocity in streamlines for locations in two dimensional system can affect the polymer adsorption, thus we expected to observe the effect of polymer adsorption during the injection time.

\section{Experimental System}

\section{Two dimensional flooding apparatus}

A flooding apparatus has been set up to conduct the two dimensional horizontal polymer flooding experiments (Figure 1). The experiment was conducted in a quarter of 5-spot pattern to represent real field problem such as viscous fingering and channeling by applying injection methods. The main components of the flooding apparatus are cylindrical plate holder for two dimensional slab (core holder) (Figure 2), fluid storage vessels, displacement pumps, effluent collector, and data acquisition system.

\section{Sandstone plate sample}

For the two dimensional flow experiments, sister gray berea sandstone slab in dimension of $20 * 20 * 2 \mathrm{~cm}$ was used. Their properties were measured to be $20.1 \%$ of porosity and $41.2 \mathrm{mD}$ of permeability. The system permeability was measured by injection of brine in direct line drive with all the open ports and calculated according to the Darcy flow equation [9]. The plate samples were washed and dried in an oven at $100^{\circ} \mathrm{C}$ for 24 hours and saturated with brine and oil to the target initial experiment condition [10].

\section{Fluids}

The oil used in this study is Shell Morlina S2BL 10 which has $10 \mathrm{cSt}$ at $40^{\circ} \mathrm{C}$. And the synthetic model of $2 \%$ salinity brines were applied in the system using 83 wt.- $\% \mathrm{NaCl}$ and 17 wt.- $\% \mathrm{CaCl}_{2}$ from

${ }^{*}$ Corresponding author: Wonmo Sung, Department of Natural Resources and Environmental Engineering, Hanyang University, Seoul, Republic of Korea, Tel: 822-2220-0414; E-mail: wmsung@hanyang.ac.kr

Received May 24, 2014; Accepted July 25, 2014; Published August 01, 2014

Citation: Park H, Han J, Lee M, Jang Y, Sung W (2014) Experimental Investigation of Polymer Adsorption-Induced Permeability Reduction in Low Permeability Reservoirs. J Pet Environ Biotechnol 5: 183. doi:10.4172/2157-7463.1000183

Copyright: $\odot 2014$ Park H, et al. This is an open-access article distributed under the terms of the Creative Commons Attribution License, which permits unrestricted use, distribution, and reproduction in any medium, provided the original author and source are credited. 


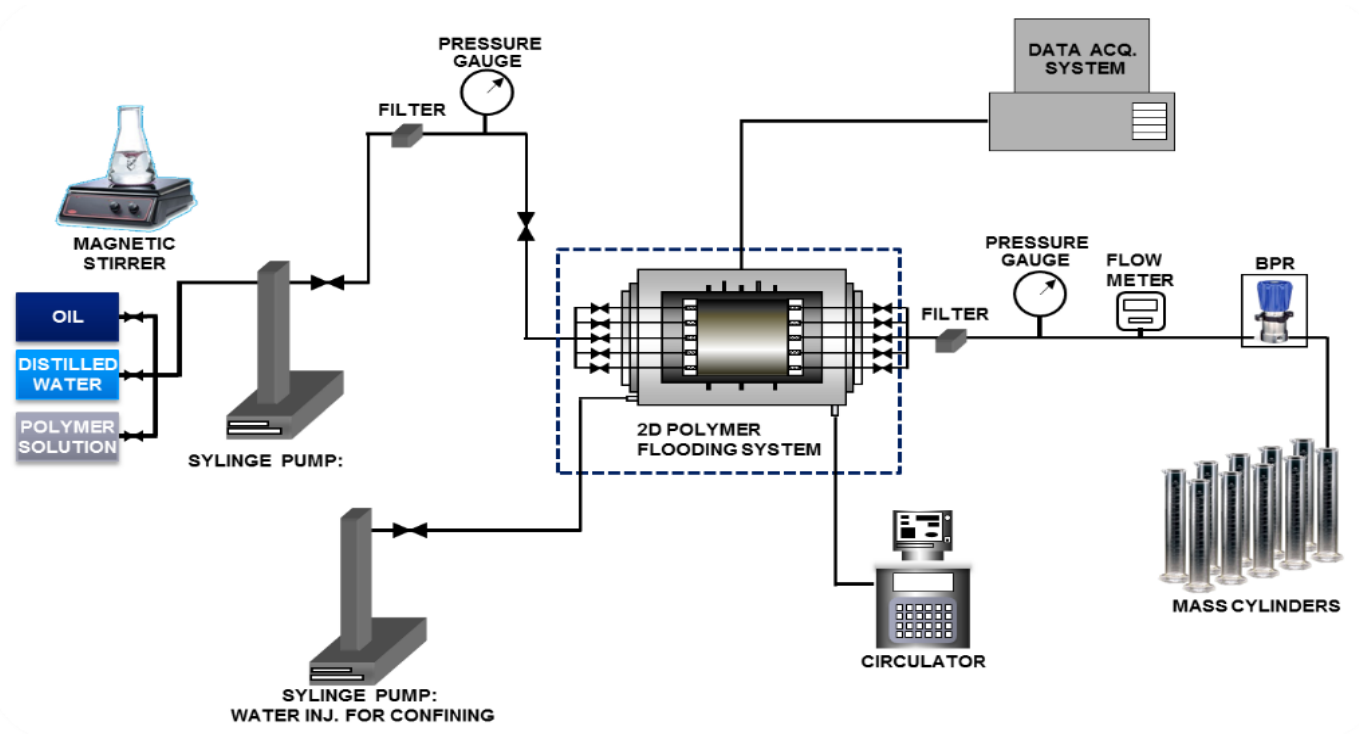

Figure 1: Schematic of polymer flooding system.

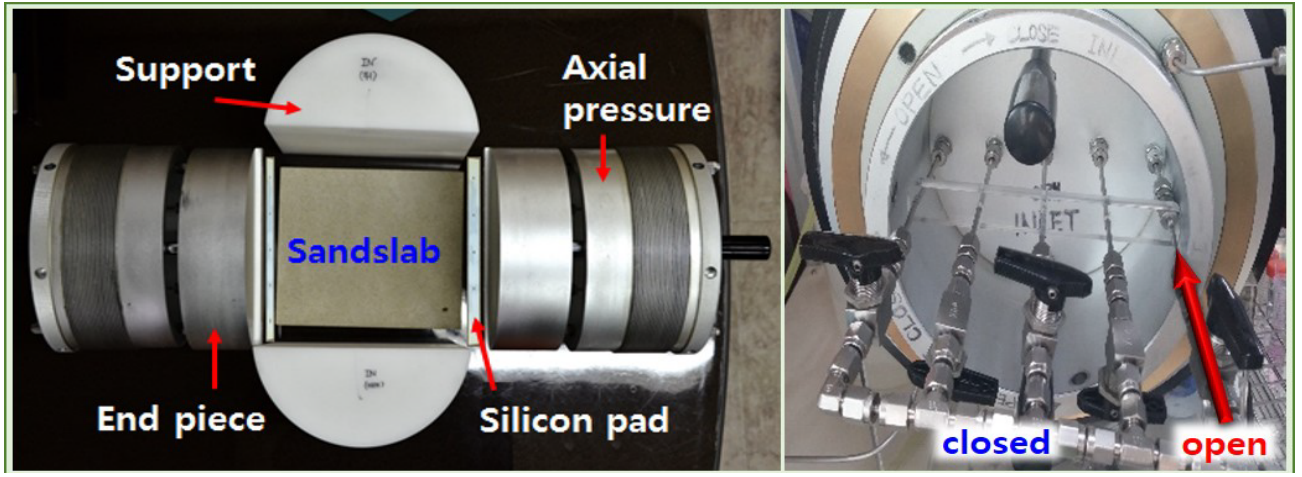

Figure 2: Two dimensional flow apparatus and opening port for a quarter of 5-spot pattern.

Winlab, UK. All experiments in this study were performed with HPAM. The polymer 3330s with molecular weight of 8 million darton was provided by SNF and was used as received in dry powder form. Polymer solutions were prepared by dissolving the required amount of polymer in $2 \% \mathrm{NaCl}$ brine, according to the procedure described in the "American Petroleum Institute (API) Recommended Practice 63 ". Stock solutions of polymer at 5,000 ppm by weight were prepared. Lower concentrations were diluted from the stock solution with the required amount of make-up brine. Polymer solutions were prepared by the slow addition of the polymer powder to the brine in a vortex created by a magnetic stirrer, gentle agitation was maintained overnight for complete polymer dissolution. To remove all microgels, the polymer solution was filtered to have lower filtration ratio than 1.2.

\section{Results and Discussion}

Firstly, the experiments have been conducted to examine the effect of polymer concentration on oil recovery by injecting $0 \mathrm{ppm}$ (brine) and $1,000 \mathrm{ppm}$ polymer solution which had $0.91 \mathrm{cp}$ and $3.42 \mathrm{cp}$ at shear rate of $100 \mathrm{~s}^{-1}$, respectively. All fluids were injected at $0.124 \mathrm{ml} /$ min which is $1 \mathrm{ft} /$ day of frontal velocity. We investigated the EOR effect corresponding to polymer concentration by considering the polymer adsorption onto the grain surface and the entrapment of polymer in the low permeability system.

The oil recovery measured for the case of $0 \mathrm{ppm}$ of polymer solution (brine) injection showed that oil was barely produced after 20.1 hours (0.96 PV) of fluid injected as water-cut increased to $99 \%$. In the case of $1,000 \mathrm{ppm}$ solution injection, water-cut maintained to be $5 \%$ to $10 \%$ which is less than $0 \mathrm{ppm}$ injection case, and oil recovery steadily increased during 46.7 hours (2.17 PV) injection and the ultimate recovery was 1.9 times higher in comparison as shown in Figures 3-5. The pressure data measured at injection and production port indicated that the pressure difference between two ports was stable for the $0 \mathrm{ppm}$ case. On the other hand, in the case of $1,000 \mathrm{ppm}$, injected polymer adsorbed onto the grain surface and was entrapped in the pore structure. That polymer retention caused the continuous increase in the injection pressure and reached to the maximum pressure at $2.17 \mathrm{PV}$ injections. The collected pressure data was used to yield the effective permeability of polymer solution by the Darcy flow equation. For both cases, after solutions were injected for 67 hours, the effective permeability of $1,000 \mathrm{ppm}$ polymer solution was calculated to be $1.05 \mathrm{mD}$ which was relatively lower than $0 \mathrm{ppm}$ having $4.36 \mathrm{mD}$. According to the results, it was figured out that the effective permeability of injection fluid 


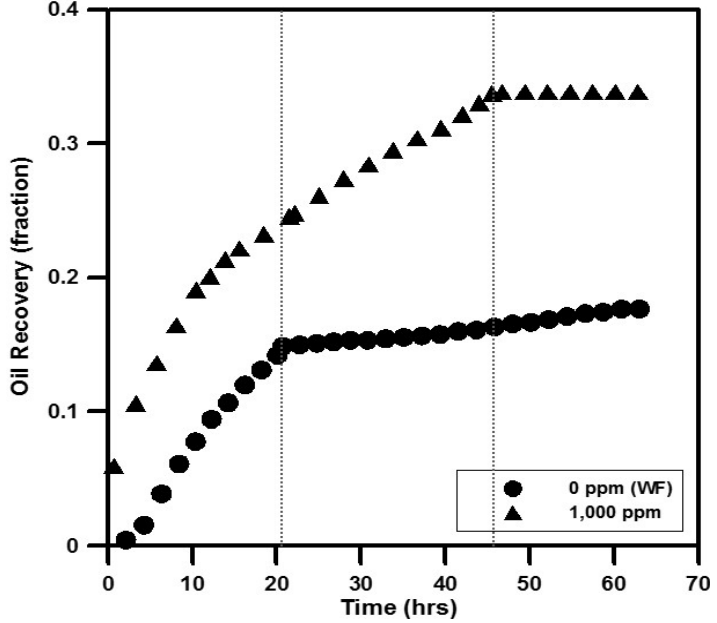

Figure 3: Oil recovery from polymer flooding with concentrations.

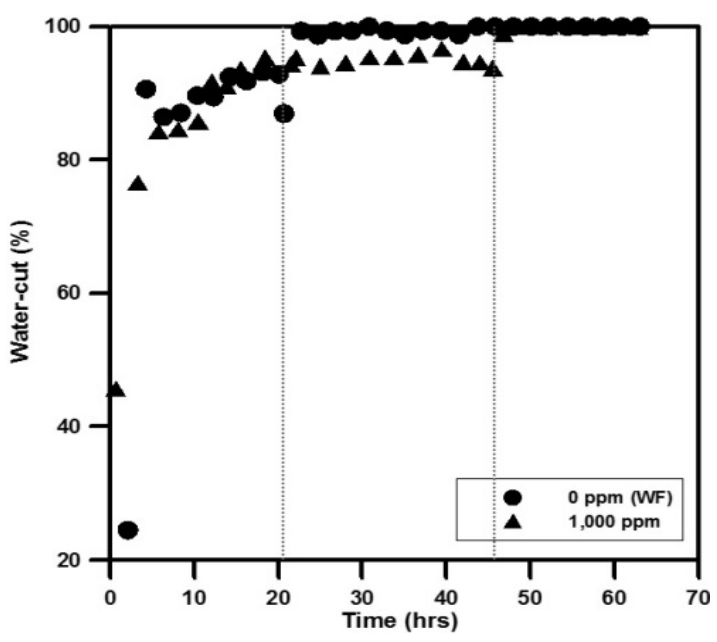

Figure 4: Water-cut results from polymer flooding with concentrations Water-cut results from polymer flooding with concentrations.

decreased more for the higher polymer concentration and when the effective permeability reduced to lower, it facilitated the oil flow and the stage of water-cut reaching 99\% was slackened off 26.6 hours (1.2 PV).

Then, we calculated the permeability reduction by polymer adsorption using the pressure data measured during the brine injection after polymer flooding. We assumed that the remaining polymer solution inside the pore including entrapped polymer except the adsorbed polymer could be removed by injecting the sufficient amount of brine after polymer flooding. When brine was injected after finishing the 1,000 ppm polymer flooding, the pressure difference between the injection and production ports dropped from 72.5 psi to 13.6 psi as shown in Figure 6. The brine permeability was computed to be $1.6 \mathrm{mD}$, which is a huge drop compared to the initial brine permeability of $41.2 \mathrm{mD}$. This result can be comprehended that the injected polymer molecular adsorbed onto the grain surface and reduced the size of the pore throat. To recapitulate, it is a fact that the polymer adsorption made the system permeability reduced, however, the system pressure were under the maximum allowable pressure though the injection pressure increased due to the polymer adsorption and entrapment during polymer flooding with 1,000 ppm solution. Also, the effective permeability of injection fluid also reduced during the flooding which affected the effluent having lowered water-cut and the effective permeability of oil became favorable so the oil recovery was enhanced. Therefore, application of polymer flooding to low permeability reservoirs can reduce the permeability even lower but still it enhances the oil recovery and decreases water production with proper concentration.

\section{Summary and Conclusion}

We conducted the experiment to investigate permeability reduction by polymer adsorption during polymer flooding particularly in low permeability reservoirs. The results showed that the ultimate oil recovery was 1.9 times higher in concentration of $1,000 \mathrm{ppm}$ case than $0 \mathrm{ppm}$ (brine). The polymer adsorption layer formed during the flooding reduced the effective permeability of $1,000 \mathrm{ppm}$ polymer solution. Therefore, it facilitated the oil flow and the stage of water-cut reaching $99 \%$ was slackened off.

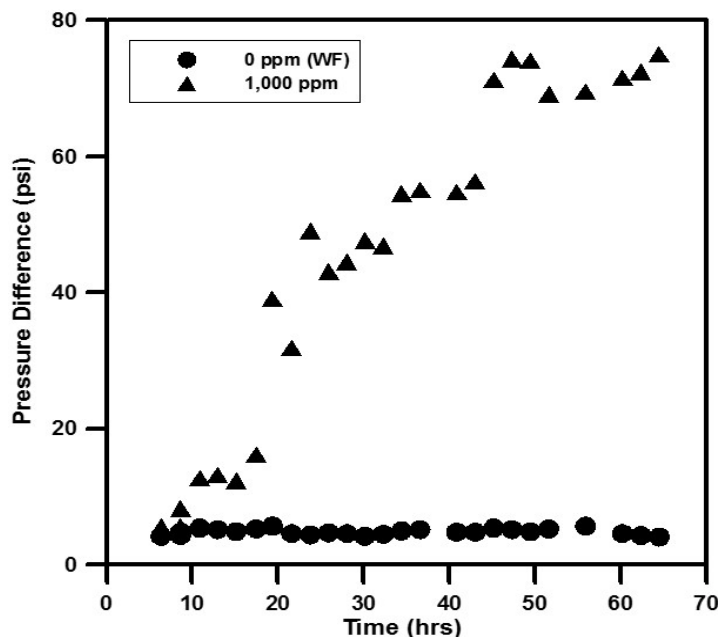

Figure 5: Pressure difference between injection and production port during polymer flooding.

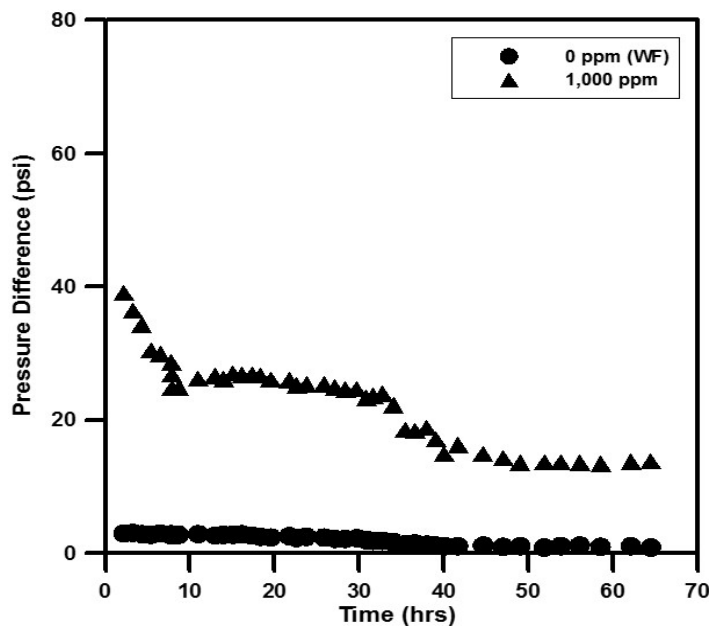

Figure 6: Pressure difference between injection and production por during brine injection after polymer flooding. 
Citation: Park H, Han J, Lee M, Jang Y, Sung W (2014) Experimental Investigation of Polymer Adsorption-Induced Permeability Reduction in Low Permeability Reservoirs. J Pet Environ Biotechnol 5: 183. doi:10.4172/2157-7463.1000183

Page 4 of 4

\section{Acknowledgement}

This work was supported by the Energy Efficiency \& Resources of the Korea Institute of Energy Technology Evaluation and Planning (KETEP) grant funded by the Korea Government Ministry of Knowledge Economy. (No. 20122010300020).

\section{References}

1. Zaitoun A, Kohler N (1988) Two-Phase Flow Through Porous Media: Effect of an Adsorbed Polymer Layer. SPE Annual Technical Conference and Exhibition, 2-5 October, Houston, Texas, USA.

2. Zaitoun A, Bertin H, Lasseux D (1988) Two-Phase Flow Property Modifications by Polymer Adsorption. Society of Petroleum Engineers.

3. Liang JT, Sun H, Seright RS (1995) Why Do Gels Reduce Water Permeability More Than Oil Permeability? Soc Petrol Eng J 10: 282-286.

4. Lee T, Jang $Y$, Park H, Lee KS, Sung W (2011) Investigation of Water BT phenomenon in the Fractured Basement Reservoir Contacted with Bottom Water Aquifer. SPE Asia Pacific Oil and Gas Conference and Exhibition, 20-22 September, Jakarta, Indonesia.
5. Lee WC, Lee YS, Kim KH, Lee KJ, Sung WM, et al. (2011) Investigation of gas and water coning behavior for the enhancement of oil production. Korean $J$ Chem Eng 28: 2102-2109.

6. Cordova M, Mogollon JL, Molero H, Navas M (2002) Sorbed Polyacrylamides: Selective Permeability Parameters Using Surface Techniques. SPE/DOE Improved Oil Recovery Symposium, 13-17 April, Tulsa, Oklahoma.

7. Barreau P, Bertin H, Lasseux D, Glénat P, Zaitoun A (1997) Water Control in Producing Wells: Influence of an Adsorbed-Polymer Layer on Relative Permeabilities and Capillary Pressure. Soc Petrol Eng J 12: 234-239.

8. Chauveteau G, Denys K, Zaitoun A (2002) New Insight on Polymer Adsorption Under High Flow Rates. SPE/DOE Improved Oil Recovery Symposium, 13-17 April, Tulsa, Oklahoma.

9. Schneider FN, Owens WW (1982) Steady-State Measurements of Relative Permeability for Polymer/Oil Systems. Soc Petrol Eng J 22: 79-86.

10. Sung W, Kang H (2003) Experimental Investigation of Production Behaviors of Methane Hydrate Saturated in Porous Rock. Energ Source 25: 845-856. 